\title{
Body weight and food intake rhythms and their relationship to the behavior of cats with brain stem lesions'
}

\author{
WALTER RANDALL AND VIRGINIA LAKSO, DEPARTMENT \\ OF PSYCHOLOGY, UNIVERSITY OF IOWA, IOWa City, Ia. \\ 52240
}

The body weight and food intake rhythms are described in normal and brain stem lesioned cats. The rhythms are similar in both groups. The lesioned group repeatedly reverts between two behavioral states, and these reversions are related to the direction of rhythmic change.

After brain stem lesions cats spontaneously and repeatedly revert between two behavioral states: the normal state and a "grooming state." In the grooming state isolated fragments of grooming behavior are elicited when light tactile stimuli are applied to the cat's body surface. These grooming fragments are consummatory behaviors which occur in complete isolation from the normally preceding orienting behaviors. The brain stem lesion, then, produces a dissociation between consummatory and appetitive behaviors (Randall, 1964). The occurrence of this abnormal dissociation is periodic, having no relationship to the time of the lesion but rather related to the spontaneous and rhythmic fluctuations in thyroid activity (Randall \& Liittschwager, 1967).

In order to adequately specify rhythmic fluctuations in thyroid activity or any other variable, repeated measures at equal intervals on the same individuals are required. Repeated measures of body weight and food intake on the same cats are easily obtainable and offer a precision that is difficult to obtain with chronic measures of thyroid activity. Body weight and food intake have been found to vary concomitantly with thyroid activity (e.g., Donhoffer \& Vonotzky, 1947). Therefore, body weight and food intake data were collected for a year in a group of normal and brain stem lesioned cats to determine with greater precision the presence of the rhythms and their relationship to the waxing and waning grooming state in cats with brain stem lesions.

Method. A year's data of body weight and food intake were obtained on ten male cats. Six of these cats are normal, and four have bilateral stereotaxic lesions in the ventral lateral portions of the brain stem at approximately the level of the lateral lemniscus. The lesions are similar to those previously illustrated (Randall \& Liittschwager, 1967).

The collection of the body weight and food intake data began at the same time for all cats a time when the lesioned group had recovered from the acute effects of the surgery. The cats were weighed every two weeks on Saturdays at approximately noon. The food was weighed before it was placed in the cages and the remainder weighed when it was removed. Two types of food constituted the main diet of the cats: (1) Friskies dry cat food which was continuously available, and (2) Friskies canned dog food which was available five days a week (Monday through Friday) from about noon to 5:00 PM. The caloric intake for two-week periods was calculated using the caloric values of the two types of food supplied by the Carnation Company. Small quantities of three special meals were provided each month in place of the canned food: raw pork liver, cooked chicken, and a milk and egg mixture. The caloric contribution of these special meals, which were fed on $10 \%$ of the days, is not considered here.

A Fourier series was fitted to the 26 measures of body weight and food intake, and differences between the fitted and observed values were evaluated with an analysis of variance model developed by Bliss (1958). One variation in Bliss's method was necessary in the present case because significant linear and quadratic trends existed in the body weight and food intake data. Thus the following composite polynomial, consisting of both algebraic and trigonometric fitting functions, was employed:

$$
\begin{aligned}
y_{t}= & a_{o}+a_{1} t+a_{2} t^{2}+a_{3} \cos \frac{2 \pi l t}{k}+a_{4} \sin \frac{2 \pi 1 t}{k}+a_{5} \cos \frac{2 \pi 2 t}{k}+ \\
& a_{6} \sin \frac{2 \pi 2 t}{k}+\ldots \ldots \ldots \ldots+a_{2 k}+3 \cos \frac{2 \pi k t}{k}+ \\
& a_{2 k}+4 \sin \frac{2 \pi k t}{k},
\end{aligned}
$$

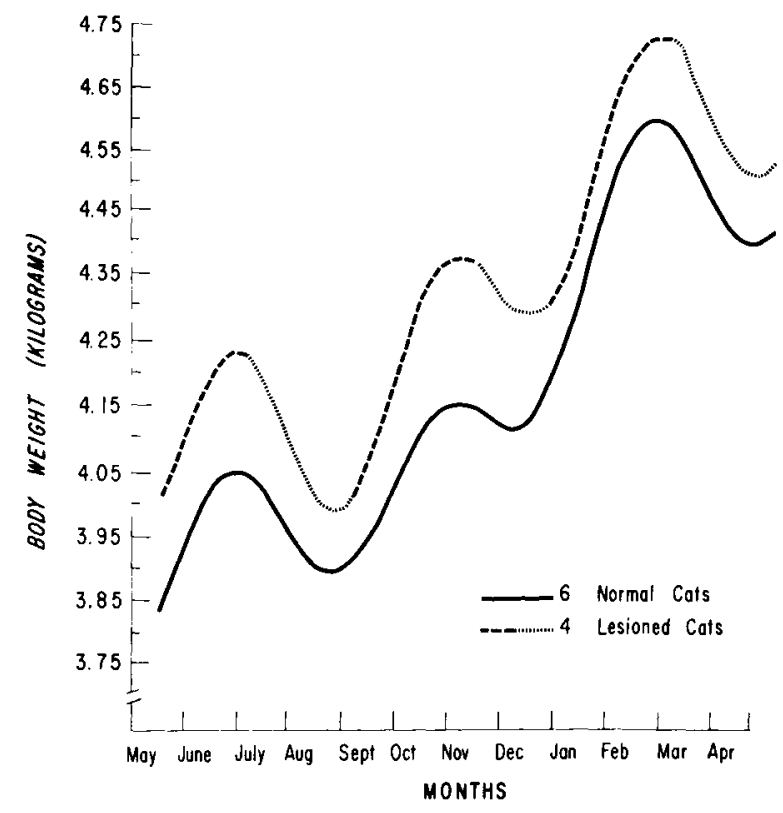

Fig. 1. The curves fitted to the body weight data with least-squares criterion. See Table 1 for the terms of the polynomials. Onsets of the grooming state occurred where the curve is dashed, and reversions to normal occurred where the curve is dotted.

where $y_{t}$ is the derived value for time $t, k$ the number of observations and $a$ the coefficients that are derived with least-squares criterion. A computer program was designed to provide the coefficients and the analysis of variance for each addition of a sine function to the series.

The grooming abnormalities were monitored as previously described (Randall, 1964; Randall \& Liittschwager, 1967), i.e., the body surface was stimulated with light tactile stimulation, and the elicited responses were recorded.

Table 1

A Summary of the Results of the Curve-fitting Procedure and the Analyses of Variance on the Body Weight Data

\begin{tabular}{lccccc}
$\begin{array}{c}\text { Terms of the } \\
\text { Polynomial }\end{array}$ & $\begin{array}{c}\text { Value of } \\
\text { coefficient }\end{array}$ & $\begin{array}{c}\text { Results of } \\
\text { F-test }\end{array}$ & & $\begin{array}{c}\text { Lesioned Group } \\
\text { Calue of } \\
\text { coefficient }\end{array}$ & $\begin{array}{c}\text { Results of } \\
\text { F-test }\end{array}$ \\
\hline $\mathrm{a}_{0}+$ & 3.828 & & 4.013 & \\
$\mathrm{a}_{1} \mathrm{t}$ & .025 & $\mathrm{p}<.005$ & .023 & $\mathrm{p}<.05$ \\
$\mathrm{a}_{3} \cos \frac{2 \pi \mathrm{t}}{26}+$ & .063 & & .051 & \\
$\mathrm{a}_{4} \sin \frac{2 \pi \mathrm{t}}{26}$ & -.062 & N.S. & -.064 & N.S. \\
$\mathrm{a}_{5} \cos \frac{4 \pi \mathrm{t}}{26}+$ & -.050 & & -.017 & \\
$\mathrm{a}_{6} \sin \frac{4 \pi \mathrm{t}}{26}$ & -.002 & N.S. & .010 & N.S. \\
$\mathrm{a}_{7} \cos \frac{6 \pi \mathrm{t}}{26}+$ & -.093 & & -.130 & \\
$\mathrm{a}_{8} \sin \frac{6 \pi \mathrm{t}}{26}$ & .050 & $\mathrm{p}<.005$ & .034 & $\mathrm{p}<.005$ \\
\hline
\end{tabular}




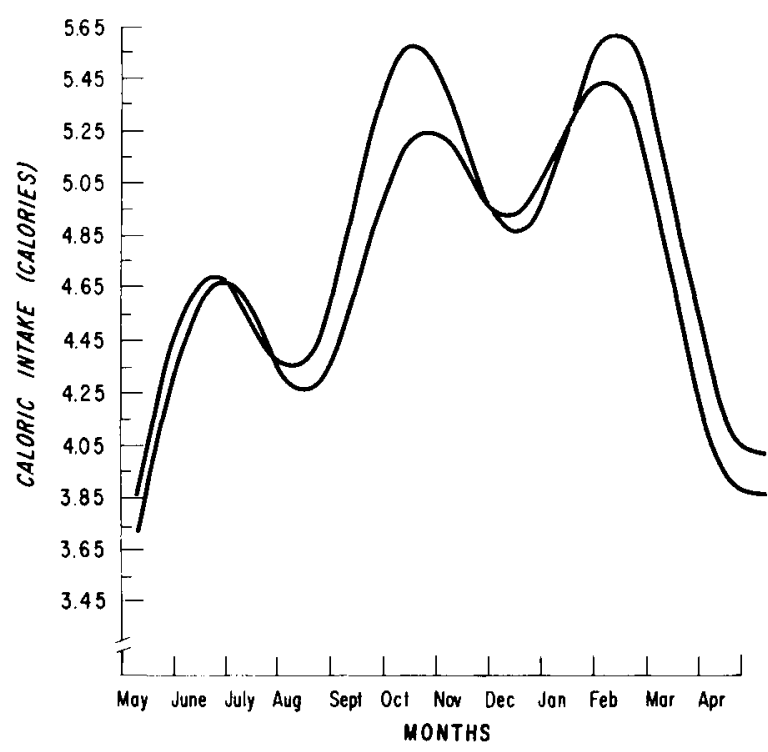

Fig. 2. The curves fitted to the caloric intake data (large calories) for both normal and lesioned groups. The lesioned group is represented by the upper curve.

Results and Discussion. Figure 1 shows the curves that were fitted to the body weight data for both normal and lesioned groups. In both groups significant linear trends existed, and, in addition, three sine functions (the annual harmonic and two subharmonics) were required to account for the periodic trend. Table 1 lists the values of the coefficients of the polynomials and the results of the analysis of variance. An F-test was made on the reduction in sum of squares attributable to the individual pairs of terms in the polynomials, and the associated p-values are listed in Table 1. A p-value less than .05 was considered significant. Each cosine-sine pair with the same multiples of $t$ constitute one sine function and are thus tested together. As indicated in Table 1, the major periodic trend is accounted for by the third harmonic, which has a duration of four months.

The body weight curve for the lesioned group is dashed and dotted to show how the reversals between the normal states and grooming states are confined to different parts of the curve. Onsets of the grooming state were confined to ascending portions or peaks of the curve (dashed portion) whereas reversions to the normal state were confined to descending portions or troughs of the curve (dotted portion). Although there were individual differences in phase angle and amplitude among the four lesioned cats, all onsets of the grooming state and all reversions to normal were segregated on their individual curves as illustrated in Fig. 1. During the year, 11 onsets of the grooming state and seven reversions to the normal state occurred for the four lesioned cats. Some of the lesioned cats on some occasions maintained the grooming state throughout descending and trough portions of the curve, a result identical to previous findings on the thyroid concomitancies.

Figure 2, representing $100 \%$ of the dry food plus about $90 \%$ of the "wet" food that was ingested, illustrates the expected similarity in caloric intake and body weight. Mathematical and statistical properties of the caloric intake curve and body weight curve are similar except for a significant quadratic trend for the caloric intake data.

Thus the presence of an approximately four-month rhythm seems well-established: both food intake and body weight in two groups of cats exhibit these rhythms which are similar in frequency to the rhythms found previously in thyroid activity in two other groups. The pronounced similarity between the rhythms of the normal and lesioned groups suggests that the nature of the lesion-effect is unrelated to the production and control of these rhythmic processes. Rather, an effect of the normal rhythmic changes on the lesioned brain is indicated because of the confinement of the two changes of behavioral state to two separate portions of the curve. All three variables (thyroid activity, food intake, and body weight) that have been selected for longitudinal study have exhibited these long term rhythms; therefore, other metabolic and endocrine variables may be predicted to exhibit these rhythms also. The onset of the grooming state may be functionally related to the effects on the lesioned nervous system of a normal, rhythmic change of one of these variables.

\section{REFERENCES}

BLISS, C. Periodic regression in biology and climatology. 1958, The Connecticut Agricultural Experimental Station, Bull. 615.

DONHOFFER, S., \& VONOTZKY, J. The effect of thyroxine on food intake and selection. Amer. J. Physiol., 1947, 150, 334-339.

RANDALL, W. The behavior of cats (Felis catus $L$.) with lesions in the caudal midbrain region. Behaviour, 1964, 23, 107-139.

RANDALL, W., \& LIITTSCHWAGER, J. The relationship between cyclic changes in thyroid function and behavior of cats with brain stem lesions. $J$. Psychiat. Res., 1967, 5, 39-58.

\section{NOTE}

1. This research was supported by USPHS Grant No. MH 12724-01. 